\title{
ADMINISTRACIÓN DEL CAPITAL DE TRABAJO, LIQUIDEZ Y RENTABILIDAD EN EL SECTOR TEXTIL DE CÚCUTA, PERIODO 2008-2011
}

\section{MANAGEMENT OF WORKING CAPITAL, LIQUIDITY AND PROFITABILITY IN THE TEXTILE SECTOR CUCUTA, PERIOD 2008-2011}

\author{
Miller Riaño-Solano ${ }^{1}$
}

Forma de citar: RIAÑO-SOLANO Miller. Administración del capital de trabajo, liquidez y rentabilidad en el sector textil de Cúcuta, periodo 2008-2011. Respuestas. 2014; 19(1): 86-98 .

Recibido:

Octubre 24 de 2013

Aceptado:

Enero 27 de 2014

86

\section{RESUMEN}

Antecedentes: Es importante destacar el sector textil como generador de empleo y divisas para una región, aunado a esto la optimización de la gestión financiera es primordial en el crecimiento y sostenibilidad de las empresas, en esa gestión es relevante el análisis del capital de trabajo, la liquidez y la rentabilidad. Objetivo: El objetivo del trabajo fue relacionar el capital de trabajo y la liquidez con la rentabilidad en el sector textil de Cúcuta en el periodo 2008-2011. Métodos: La metodología utilizada, consistió en un diseño documental, basada en una investigación descriptiva correlacional. Las fuentes, secundarias, donde se consultó la información financiera relacionada con el sector. Se tabularon los datos, y se analizaron usando técnicas estadísticas para relacionar el coeficiente de correlación (CC) y el coeficiente de determinación (CD). Resultados: Las variaciones del Capital de Trabajo Neto Operativo (KTNO) explicaron en un 89,4\% las variaciones de la Rentabilidad del Activo Neto (RAN) y en un 98,1\% las variaciones de la Rentabilidad del Patrimonio (ROE). Las Cuentas por Cobrar (CxC) representaron el 96,67\% de las variaciones del ROE, los inventarios lo hicieron en un 76,89\% y las Cuentas por Pagar (CxP) en el 95,26\%. La liquidez fue la variable de menor influencia en las variaciones del ROE. Conclusión: Los resultados obtenidos se alinearon con los postulados teóricos de Gitman, Albornoz y García. En general, se aprecia la importancia de la adecuada gestión del KTNO como factor de criticidad en la gestión operativa del sector textil confecciones para este periodo, aportando información a este sector para coadyuvar a posicionar en el mercado local e internacional, avanzando en el Proyecto de Transformación Productiva Sectores de clase Mundial impulsado por el Ministerio de Comercio, Industria y Turismo.

Palabras clave: Capital de Trabajo, Rentabilidad, Liquidez, Correlación. 
Background: It is important to emphasize the textile sector as generator of employment and currencies for a region, in addition to this the optimization of the financial management is essential in the growth and sustainability of the companies, management is relevant in that the analysis of working capital, liquidity and profitability. Objective: The objective was to relate working capital and liquidity to profitability in the textile sector of Cucuta in 20082011. Methods: The methodology, design consisted of a documentary based on a descriptive correlational research. The secondary sources where the financial information relating to the sector were consulted. The data were tabulated and analyzed using statistical techniques to relate the correlation coefficient and the coefficient of determination. Results: Changes in Net Operating Working Capital explained $89.4 \%$ variation of Return of Net Assets and 98.1\% variations in Return on Equity (ROE). Accounts Receivable accounted for $96.67 \%$ of the variation in ROE, inventories grew by $76.89 \%$ and Accounts Payable at $95.26 \%$. The liquidity was the variable of less influence in the changes of the ROE. Conclusion: The results obtained were aligned with the theoretical postulates of Gitman, Albornoz and Garcia. In general, the importance of proper management of working capital seen as critical factor in the operational management of the textile confections this period, providing information to assist this sector to position in the local and international market, advancing in the Draft Productive Transformation Sectors world class launched by the Ministry of Commerce, Industry and Tourism.

Keywords: Working Capital, Profitability, Liquidity, Correlation.

\section{INTRODUCCIÓN}

E n las finanzas es importante el estudio del Capital de Trabajo. Es una etapa indispensable del análisis financiero. A continuación se precisan algunas definiciones para el desarrollo del tema. García (2003) expone tres clasificaciones para el concepto: Capital de Trabajo (KT), Capital de Trabajo Operativo (KTO) y Capital de Trabajo Neto Operativo (KTNO). El autor define Capital de Trabajo como los recursos que una empresa requiere para llevar a cabo sus operaciones sin contratiempos, mientras que el Capital de Trabajo Operativo está representado solo por la suma de las cuentas por cobrar y los inventarios y agrega que el Capital de Trabajo Neto Operativo está representado por el neto entre las cuentas por cobrar más los inventarios menos las cuentas por pagar a proveedores de bienes y servicios.

Los pilares en que se basa se sustentan en la medida en que se pueda hacer un buen manejo sobre el nivel de liquidez. Mientras más amplio sea el margen entre los activos corrientes que posee la organización y sus pasivos, más amplio será la capacidad de cubrir las obligaciones a corto plazo, sin embargo, se presenta un gran inconveniente porque cuando exista un grado de liquidez relacionado con cada recurso y cada obligación, al momento de no poder convertir 
No. 1

Enero - Junio 2014

ISSN 0122-820X

PP: $86-98$ los activos corrientes más líquidos en dinero, los siguientes activos tendrán que sustituirlos. Mientras más de estos se tengan mayor será la probabilidad de tomar y convertir cualquiera de ellos para cumplir con los compromisos contraídos. (Faxas y Atucha, 2011, p.1)

En tanto se mantenga el equilibrio fijado como meta, los pasivos circulantes podrán ser pagados oportunamente, los proveedores continuarán embarcando bienes y reabasteciendo los inventarios y estos serán suficientes para satisfacer la demanda de venta. Sin embargo si la situación financiera se aleja del equilibrio programado, surgirán y se multiplicarán los problemas, y la empresa podrá verse envuelta en una espiral descendente que la lleve a la quiebra.

En opinión de Van Horne J. y Wachowicz, J. (1994, p. 251) la administración del capital de trabajo corresponde a "La administración de los activos circulantes de la empresa y el financiamiento que necesita para sostener activos circulantes".

Mientras que para Moyer y McGuigan (2005, p. 516) determinar el nivel óptimo de inversión en capital de trabajo comprende un análisis de las interrelaciones entre rentabilidad y riesgo, ya que mayores niveles de capital de trabajo reducen la rentabilidad y mayores niveles de capital de trabajo reducen el riesgo de dificultades financieras.

Respecto a la optimización del capital de trabajo Cabrera y Amero (2006) indican que ante el hecho de que todo está interconectado de manera causal en la organización, y que casi nada sucede sin tener un impacto positivo o negativo en la liquidez y la rentabilidad del negocio, el gestionar de manera integral estos procesos se ha convertido en uno de los principales retos para los Directores de Finanzas, pues en la mayoría de los casos estos procesos fluyen de manera aislada y asincrónica.

Como complemento a la importancia de la administración del capital de trabajo, otros autores sostienen que entre las causas de los fracasos de las empresas se encuentra tener más de lo debido tal como sobreinversión en inventarios, sobreinversión en cuentas y documentos por cobrar y también sobreinversión en activos fijos y en contraposición tener menos de lo debido por insuficiencia de ventas, insuficiencia de capital o insuficiencia de utilidades.

En este orden de ideas García (2003) señala que una empresa exitosa debe ser liquida y rentable a la vez e indica que en corto plazo la liquidez y la rentabilidad son antagónicas y expone también la relación directa que existe entre rentabilidad y riesgo. En este sentido también propone el análisis de la Productividad del Capital de Trabajo (PKT $=\mathrm{KTNO} /$ Ventas) entendida como los centavos que por cada peso de ventas deben mantenerse en Capital de Trabajo Neto Operativo (KTNO). Recomienda que lo ideal es mantener el número lo más pequeño posible.

Sobre este punto Moyer (2005) sostiene que en la política general de capital de trabajo se consideran tanto el nivel de inversión como el de financiamiento y que en la práctica se debe determinar el impacto conjunto de esas decisiones en su rentabilidad y riesgo. En su análisis Moyer expone que las políticas de capital de trabajo, dado su efecto en los rendimientos futuros esperados y el riesgo asociado con estos, tienen un importante impacto en la riqueza de los accionistas, señala también que la rentabilidad se mide de acuerdo con la tasa de rendimiento sobre activos totales (EBIT/activos totales).

Para Van Horne (2002), detrás de la administración adecuada del capital de trabajo subyacen dos aspectos fundamentales relacionados con la toma de decisiones en las empresas, referentes a la determinación del nivel óptimo de inversión en activo circulante y la mezcla adecuada de financiamiento a corto y largo plazos utilizada para apoyar esta inversión en activo circulante. Al mismo tiempo, estas decisiones se ven influidas por el equilibrio que debe existir entre la rentabilidad y el riesgo. Si se reduce el nivel de inversión en activo fijo, sin perder la capacidad de apoyar las ventas, se incrementará el rendimiento del 
activo total de las empresas. En la medida en que el costo explícito del financiamiento a corto plazo es menor que el del financiamiento a mediano y largo plazos, es mayor la proporción entre la deuda a corto plazo y la deuda total, y más alta la rentabilidad de las compañías.

En ese mismo sentido Van Horne agrega que estas premisas de rentabilidad sugieren mantener un nivel bajo de activo circulante y una elevada proporción de pasivo circulante y pasivo total e indica que esa estrategia se traducirá en un capital de trabajo neto bajo, o evidentemente negativo. No obstante, compensar la rentabilidad de esta estrategia es el riesgo mayor de las empresas. En este sentido, este último implica el peligro de no tener suficiente activo circulante para: Cumplir con sus obligaciones y mantener los niveles adecuados de ventas (por ejemplo, agotar el inventario) para ello se apoya en la relación

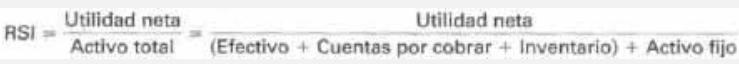

Por las consideraciones anteriores, se puede observar que con la disminución del activo se incrementará la rentabilidad potencial. $\mathrm{Si}$ se pueden reducir las inversiones de la empresa en activo circulante, sin dejar de apoyar de manera adecuada la producción y las ventas, aumentará el RSI. Los niveles bajos de efectivo, las cuentas por cobrar y el inventario reducirían el denominador de dicha ecuación; y las utilidades netas, el numerador, se mantendrían constantes, o quizás hasta registrarían un aumento.

Igualmente Gitman (2003) al estudiar la administración financiera a corto plazo, el capital de trabajo neto y la relación entre rentabilidad y riesgo señala que la administración financiera a corto plato se enfoca en administrar cada uno de los activos circulantes de la empresa (inventario, cuentas por cobrar, efectivo y valores negociables) y los pasivos circulantes (cuentas por pagar, cargos por pagar y documentos por pagar) de una manera que contribuye positivamente al valor de la empresa (p.522).
Refiere el autor que suponiendo un nivel constante de activos totales, cuanto mayor sea la razón de activos circulantes sobre activos totales, menos rentable y riesgosa es la empresa. Lo contrario también es cierto. Con los activos totales constantes, cuanto mayor es la razón de pasivos circulantes sobre activos totales de una empresa, más rentable y más riesgosa es la empresa y dice que lo opuesto también es cierto. Agrega respecto al ciclo de conversión de efectivo que la duración del ciclo determina cuánto tiempo se invierten los recursos en las operaciones cotidianas de la empresa e indica que con frecuencia, la inversión de la empresa en activos a corto plazo consta de requerimientos permanentes y estacionales de financiamiento (p. 523). Los requerimientos estacionales se pueden financiar utilizando ya sea una estrategia de financiamiento agresiva de bajo costo y alto riesgo, o una estrategia conservadora de alto costo y bajo riesgo. A fin de cuentas, la decisión de la empresa acerca de su ciclo de conversión de efectivo depende de la disposición de la administración hacia el riesgo y la fuerza de las relaciones bancarias de la empresa.

En relación a este tema Albornoz (2006) expone que la administración del capital de trabajo se ocupa de la administración de los activos corrientes (caja, bancos, cuentas a cobrar, inversiones líquidas, inventarios) como así también de los pasivos corrientes y señala que administrar tal capital se consigue buscando un equilibrio entre la liquidez y la rentabilidad dado que dichas funciones son contrapuestas entre sí. Señala, que una política conservadora en la administración de activos de trabajo traerá con ella una minimización del riesgo en oposición a la maximización de la rentabilidad. Opuestamente, una política agresiva pondría énfasis en el aspecto de los rendimientos sobre la decisión riesgo rentabilidad.

Albornoz también analiza las diferentes variables y la relación que a su juicio deberían presentarse al estudiar la relación capital de trabajo-rentabilidad-riesgo. Al respecto señala que desde el punto de vista de los activos corrientes, cuanto menor sea la proporción
Enero - Junio 2014

ISSN 0122-820X

PP: $86-98$ 
No. 1

Enero - Junio 2014

ISSN 0122-820X

PP: $86-98$ de éstos, mayor será la rentabilidad sobre el total de las inversiones de la empresa y que si se analiza desde el lado de los pasivos, la rentabilidad se mide por el costo de las distintas fuentes de financiamiento y el uso durante los períodos en que no se los utiliza. Además, dado que normalmente los costos explícitos de las deudas a corto plazo son menores que los de las de largo plazo, cuanto mayor sea la proporción del uso de las deudas de corto plazo, mayor será la rentabilidad de la empresa. Además, la utilización de deudas de corto plazo permite disminuirlas en aquellos momentos en que por estacionalidad o por incrementos en los ingresos no se necesitan; esto también hará obtener una mayor rentabilidad.

En función de lo expresado, será conveniente tener una baja proporción de activos corrientes o circulantes sobre los activos totales y una alta proporción de pasivos corrientes sobre el endeudamiento total. Esto producirá obviamente un bajo nivel de capital de trabajo.

En atención a la definición de Empresa, la Comisión de la Unión Europea sugiere la siguiente definición: "Se considerará empresa toda entidad, independientemente de su forma jurídica, que ejerza una actividad económica. En particular, se considerarán empresas las entidades que ejerzan una actividad artesanal $\mathrm{u}$ otras actividades a título individual o familiar, las sociedades de personas, y las asociaciones que ejerzan una actividad económica de forma regular".

Para Correa (2009), dentro de esta clasificación aparece uno de los sectores que a nivel mundial tiene un gran peso específico, esa es la Industria textil. Es el nombre que se da al sector de la economía dedicado a la producción de ropa, tela, hilo, fibra y productos relacionados siendo productos de consumo masivo que se venden en grandes cantidades. Agrega el autor en su trabajo que la industria textil genera gran cantidad de empleos directos e indirectos y tiene un peso importante en la economía mundial. Sostiene que es uno de los sectores industriales que más controversias generan, especialmente en la definición de tratados comerciales internacionales debido principalmente a su efecto sobre las tasas de empleo.

La investigación es importante porque apoya los objetivos planteados en el programa "Perspectivas del sector textil-confecciones 2010-2032" del Ministerio de Comercio, Industria y Turismo de Colombia. Si bien el sector textil confecciones ha enfrentado una de las peores crisis en los últimos tres años, hoy tiene la oportunidad de asegurar la posición del sector en el mercado local e internacional, avanzando en el Proyecto de Transformación Productiva Sectores de clase Mundial impulsado por el Ministerio de Comercio, Industria y turismo, el cual busca generar ingresos de al menos US $\$ 14.3$ mil millones, multiplicando el sector casi cuatro veces y generando 85.000 empleos aproximadamente al 2032.

Sobre el tema se refieren Arcos y Benavides (2006) en "Efecto del ciclo de efectivo sobre la rentabilidad de las firmas colombianas". En ese documento analizan el impacto del ciclo de efectivo sobre la rentabilidad de las empresas en Colombia para un periodo de cinco años (2001-2004). En el análisis concluyen que cuando la rentabilidad se mide sobre el nivel de ventas, se encuentra un efecto negativo entre el número de días del ciclo de efectivo y esa rentabilidad. El resultado confirma la hipótesis que asocia los ciclos de efectivo cortos con una mayor rentabilidad.

Igualmente Restrepo, M; Aníbal J; Vanegas, J. (2010). Competitividad y comercio exterior de las Pymes del sector textilconfecciones del Valle de Aburrá: incidencia del tipo de cambio. Mediante el cálculo del margen de contribución (MC). El 43\% de las empresas analizadas obtuvieron un desempeño sobresaliente, el 14\% están en vía de ser competitivas, el $12 \%$ fueron poco competitivas y el $31 \%$ no son competitivas. Las Pymes presentan un MC promedio del $26 \%$, insuficiente para cubrir los costos fijos y generar utilidad

Se referencia a Rizo L, Solís P. (2010) "La 
teoría del capital de trabajo y sus técnicas”. La magnitud del mismo depende de la actividad que realiza la empresa y de la posición que ella está dispuesta a asumir ante el riesgo de insolvencia; de esta manera mientras mayor cantidad de capital de trabajo tenga la empresa, mayor posibilidad de pagar sus deudas en el momento de su vencimiento, pero también menor rendimiento en el financiamiento. Es por eso que la magnitud del capital de trabajo que debe buscar la empresa debe ser aquella que provenga de la optimización de los inventarios, cuentas por cobrar, cuentas por pagar y del efectivo para operar.

Respecto al sector textil colombiano, en el Informe Sector Textil y Confecciones Colombiano. Análisis Sectorial, la crisis actual por la cual atraviesa la industria textil-confecciones obedece a varios factores que se conjugaron en contra del mercado colombiano en los tres últimos años, como la caída de la demanda mundial por efectos de la crisis financiera, la revaluación del peso, la baja en los precios internacionales de algunas materias primas, el contrabando como gran responsable de la crisis interna del sector y del desempleo, y el cierre de mercados clave para Colombia, como Ecuador y Venezuela, siendo estos últimos factores, los de mayor incidencia en el comportamiento del sector externo.

En lo que se refiere al sector textil en el Departamento Norte de Santander, Amaya (2009) expuso que la situación de las factorías colombianas se ha agudizado por la mora que presenta Cadivi, asunto que redunda en desempleo, incumplimientos de pago con proveedores y endeudamiento con la banca nacional para salir de líos, como les pasa a las pequeñas y medianas empresas. Añadió en su intervención que este problema está creciendo y se nos está saliendo de las manos porque CADIVI no gira y no paga las divisas correspondientes a nuestras exportaciones.

Atendiendo lo anterior, surge el interés por conocer como se relacionaron el capital de trabajo, la liquidez y la rentabilidad en el sector textil de Cúcuta en el periodo 2008-2011 y se plantea como objetivo general "Relacionar el capital de trabajo, la liquidez y la rentabilidad en el sector textil de Cúcuta periodo 2008. 2011".

\section{METODOLOGIA}

\section{Nivel y Diseño de la Investigación}

La investigación se realizó utilizando un enfoque cuantitativo tal como lo señalan Hernández, R., Fernández y Baptista (2003), el mismo se emplea cuando se recolectan y analizan datos que luego darán respuesta a las preguntas de investigación, en este caso corresponde a la información estadística que se tomó de los informes técnicos y financieros publicados sobre las PYME's del sector textil del Departamento Norte de Santander durante el periodo de estudio.

Se enmarcó en una investigación de tipo descriptiva correlacional donde se analizó la relación entre las variables sin establecer relaciones de causalidad. Tal como lo explica Ávila (2006), los estudios descriptivos son el precedente de la investigación correlacional y tienen como propósito la descripción de eventos, situaciones representativas de un fenómeno o unidad de análisis específica y señala respecto a los estudios correlaciónales que ellos tienen como objetivo medir el grado de asociación entre dos o más variables, mediante herramientas estadísticas de correlación. Indica que en este nivel no es importante el orden de presentación de las variables, lo fundamental es determinar el grado de relación o asociación existente.

Se desarrolló como a una investigación documental. La investigación documental es definida como: "un análisis de la información escrita sobre un determinado tema, con el propósito de establecer relaciones, diferencias, etapas, posturas o estado actual del conocimiento respecto al tema objeto de estudio." (Bernal, 2000, p.111).

\section{Población y Muestra}

Los criterios de inclusión para determinar la población fueron los siguientes: Empresas
Enero - Junio 2014 ISSN 0122-820X PP: 86-98 
No. 1

Enero - Junio 2014 ISSN 0122-820X

PP: 86-98 clasificadas del sector textil, establecidas en la ciudad de Cúcuta, que reporten a la Superintendencia de Sociedades. En la investigación se analizó la información financiera de las empresas reportadas por SUPERSOCIEDADES en la base de datos de SIREM

La validez y confiabilidad de la información está soportada por los registros electrónicos de la página web oficial de la Superintendencia de Sociedades (SUPERSOCIEDADES). La Superintendencia de Sociedades requiere cada año a un número determinado de empresas para la presentación de estados financieros de fin de ejercicio (31 de diciembre). Entre las empresas requeridas se encuentran las vigiladas (Decretos 4350 de 2006 y 2300 de 2008), controladas (Art. 85 de la Ley 222 de 1995) y una muestra de empresas inspeccionadas (Art. 83 de la Ley 222 de 1995).

\section{Recolección de datos.}

Las fuentes en la investigación, fueron secundarias mediante la consulta de bibliotecas (libros, revistas), documentos (diarios, periódicos), datos estadísticos, informes e información financiera del sector en estudio utilizando como técnica el registro de datos mediante el uso de unidades de almacenaje.

\section{Análisis de los datos.}

En el análisis de datos se siguió el criterio de García (2003) para el capital de trabajo neto operativo (KTNO) y los criterios de Albornozy de Gitman para el análisis de la administración del capital de trabajo y de la rentabilidad. El estudio relacionó el comportamiento de las variables KTNO, liquidez y rentabilidad de las empresas que conforman el sector textil en el municipio de San José de Cúcuta para el periodo 2008-2011.

El método estadístico aplicado para analizar la relación entre las variables, fue el análisis de correlación para determinar el coeficiente de correlación de Pearson y el coeficiente de determinación R2, lo cual permite evaluar la relación entre dos variables categóricas. A continuación se presenta un ejemplo de tabla de correlación, dado que se aplicó el mismo procedimiento para las demás variables.

Tabla 1 Resumen KTNO y Rentabilidad del Activo Neto (RAN)

\begin{tabular}{|lc|}
\hline \multicolumn{2}{|c|}{ Estadisticas de la regresión } \\
\hline Coeficiente de correlación múltiple & 0,945524002 \\
Coeficiente de determinación $\mathrm{R}^{\wedge} 2$ & 0,894015638 \\
$\mathrm{R}^{\wedge} 2$ ajustado & 0,788031276 \\
\hline
\end{tabular}

En este caso se determinó que las variables KTNO y RAN presentaron un coeficiente de correlación lineal de 0,9455 el cual indica una fuerte asociación positiva, es decir se mueven en la misma dirección, mientras que el coeficiente de determinación R2 señala que las variaciones de la RAN pueden ser explicadas en un $89,4 \%$ por las variaciones del KTNO.

\section{Análisis de los Resultados}

El análisis de la información aplicando los criterios de Albornoz, de Gitman y de García permitió determinar:

\section{Índice de liquidez y Rentabilidad del patrimonio (ROE)}

En la figura uno se muestra la relación entre el índice de liquidez y ROE,

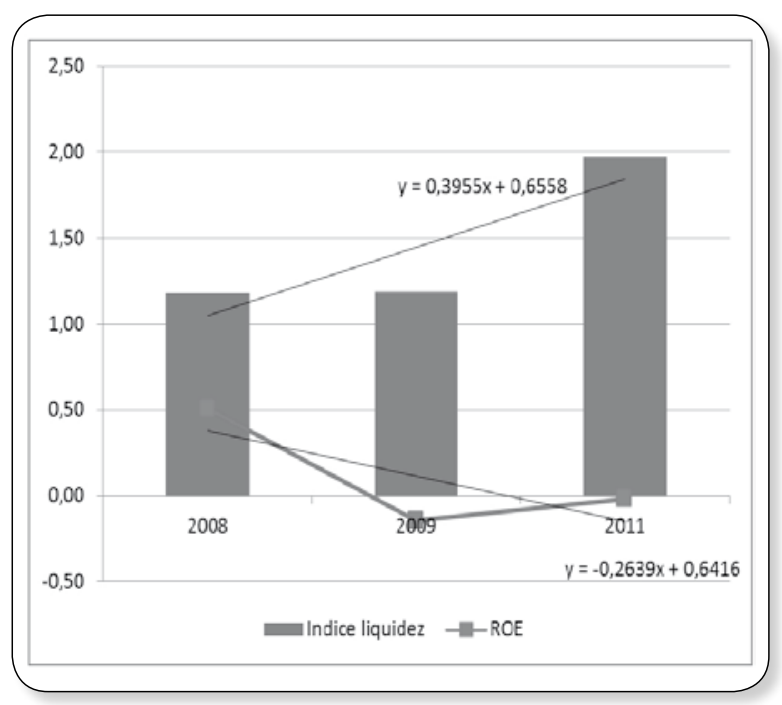

Figura 1 Relación índice de liquidez y ROE

Se observa que el índice de liquidez tiene 
una tendencia al aumento mientras que la tendencia de $\mathrm{ROE}$ es a la disminución. Esto se encuentra alineado con la teoría financiera donde se expone que una alta liquidez tiende a desmejorar la rentabilidad.

\section{Relación activo corriente/activo total; pasivo corriente/pasivo total y $\mathrm{ROE}$}

La relación entre el activo corriente/activo total; pasivo corriente/pasivo total y ROE se presenta en la figura dos.

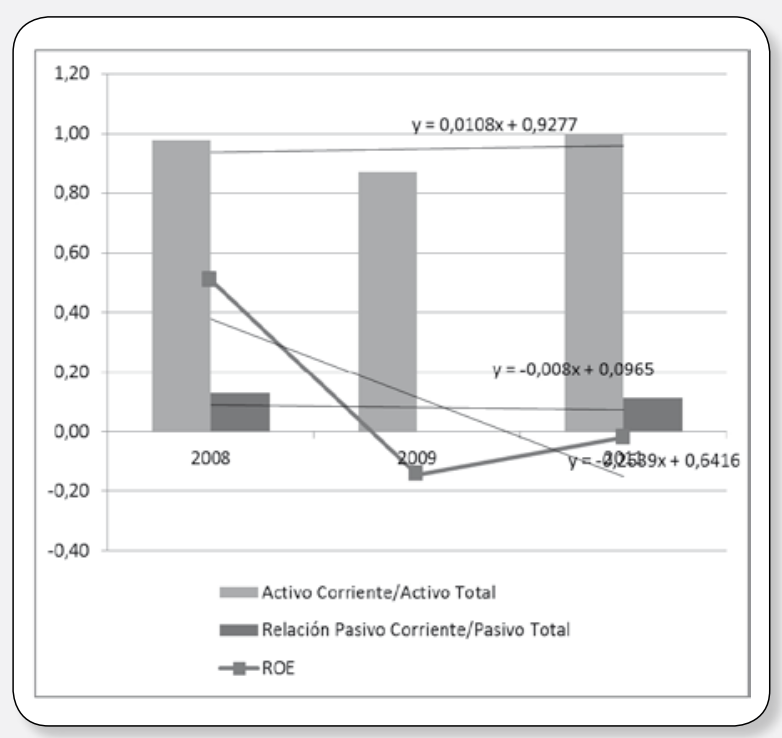

Figura 2. Relación activo corriente/activo total y ROE

En la relación activo corriente/activo total; pasivo corriente/pasivo total y ROE, no se aprecia un completo ajuste a la teoría financiera, en ella se plantea que a baja relación activo corriente/activo total, se tiene mayor rentabilidad y además que una alta relación pasivo corriente/pasivo total, conlleva a mayor rentabilidad. No obstante se observa que la rentabilidad mejoró a partir del año 2009 ante una variación del 2,2 \% de la relación activo corriente/activo total, mientras que la relación pasivo corriente/ pasivo total varió en 0,02 puntos lo que podría dar un indicio de alineación a la teoría.
Relación PKT y ROE

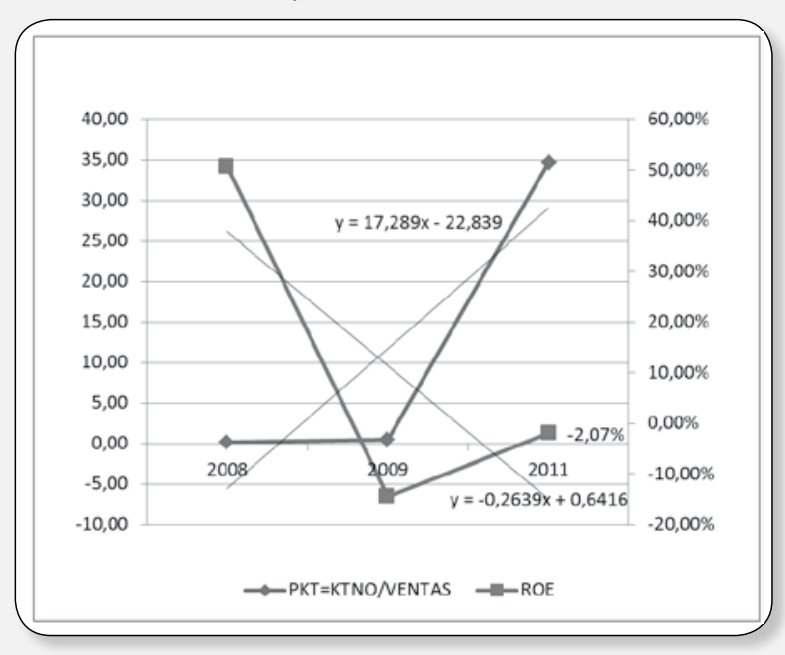

Figura 3. Relación PKT y ROE

Según García, si PKT tiende a cero hace más eficiente la administración del KTNO y por ende podría lograrse mayor rentabilidad. En este caso se observa concordancia entre los resultados y la teoría a pesar del comportamiento del año 2011 donde se aprecia desmejora en el índice PKT y una mejora en ROE aunque continúa siendo negativa, notándose en el periodo de estudio que la tendencia de PKT es al incremento y la de $\mathrm{ROE}$ es a la desmejora.

\section{Relación ciclo de efectivo y ROE}

La figura cinco muestra la relación ciclo de efectivo y ROE

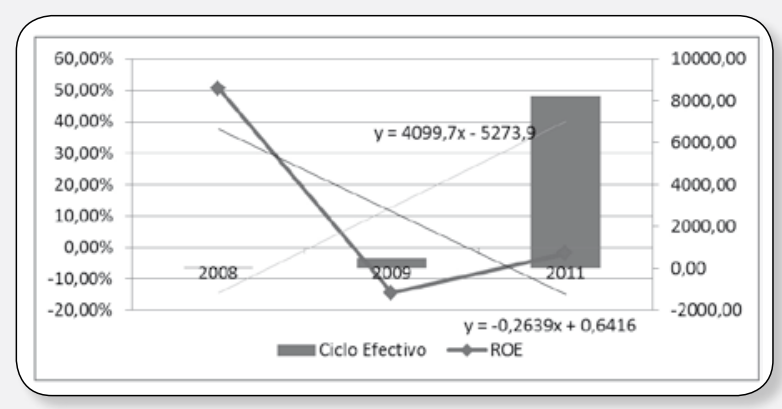

Figura 4. Relación ciclo de efectivo y ROE

Se observa que el aumento del ciclo de efectivo estuvo acompañado de la desmejora de $\mathrm{ROE}$, tal como se plantea en la teoría financiera.

A continuación se presentan los análisis de correlación para medir la relación de cada una 
No. 1

Enero - Junio 2014 ISSN 0122-820X

PP: 86-98 de las variables.

Los cálculos se efectuaron utilizando la hoja de cálculo Excel de Microsoft ${ }^{\circledR}$, los resultados se presentan en las tabla 2 (Calculo del Coeficiente de Correlación de Pearson) y en la tabla 3 (Calculo del Coeficiente de determinación R2)

Tabla 2. Calculo del Coeficiente de Correlación de Pearson

\begin{tabular}{lrrrrrr}
\hline & KTNO & LIQUIDEZ & $F C L$ & RAN & ROE & MEBITDA \\
\hline KTNO & 1 & \multicolumn{1}{c}{1} & & & & \\
LIQUIDEZ & $-0,467827194$ & 1 & 1 & & & \\
FCL & $-0,455477281$ & $-0,573733768$ & 1 & & \\
RAN & 0,945524002 & $-0,154612137$ & $-0,720486877$ & 1 & & \\
ROE & 0,990313358 & $-0,340576704$ & $-0,574676505$ & 0,981568152 & 1 & \\
MEBITDA & 0,40373265 & $-0,997463674$ & 0,630575839 & 0,083898508 & 0,272790734 & 1 \\
\hline
\end{tabular}

Al calcular el coeficiente de correlación lineal de Pearson se encontró que el KTNO y el ROE presentaron un coeficiente de correlación de 0,9903 que se puede considerar muy alto positivo y con el RAN de 0,9455 al cual se le puede dar el mismo tratamiento. Esto significa que a medida que varía uno de los factores el otro se mueve en la misma dirección y están fuertemente asociados. Se podría explicar esto porque a medida que varía el KTNO hace que los recursos disponibles aumenten o disminuyen afectando los beneficios y la rentabilidad.

Tabla 3. Calculo del Coeficiente de determinación R2

\begin{tabular}{lrrrrrr}
\hline & KTNO & LIQUIDEZ & \multicolumn{1}{c}{$F C L$} & \multicolumn{1}{c}{ RAN } & ROE & MEBITDA \\
\hline KTNO & $100,0 \%$ & & & & & \\
LIQUTDEZ & $21,9 \%$ & $100,0 \%$ & & & & \\
FCL & $20,7 \%$ & $32,9 \%$ & $100,0 \%$ & & & \\
RAN & $89,4 \%$ & $2,4 \%$ & $51,9 \%$ & $100,0 \%$ & & \\
ROE & $98,1 \%$ & $11,6 \%$ & $33,0 \%$ & $96,3 \%$ & $100,0 \%$ & \\
MEBITDA & $16,3 \%$ & $99,5 \%$ & $39,8 \%$ & $0,7 \%$ & $7,4 \%$ & $100,0 \%$ \\
\hline
\end{tabular}

Se determinó que las variaciones del KTNO explican en un $89,4 \%$ las variaciones de la

94 RAN y en un $98,1 \%$ las variaciones del ROE, es decir se estableció la importancia de la adecuada gestión del KTNO como factor de criticidad en la gestión operativa del sector textil confecciones.

Se analizó la relación entre los componentes del KTNO por separado con el ROE. En la figura cinco se presenta la relación entre $\mathrm{CxC}$ y el ROE

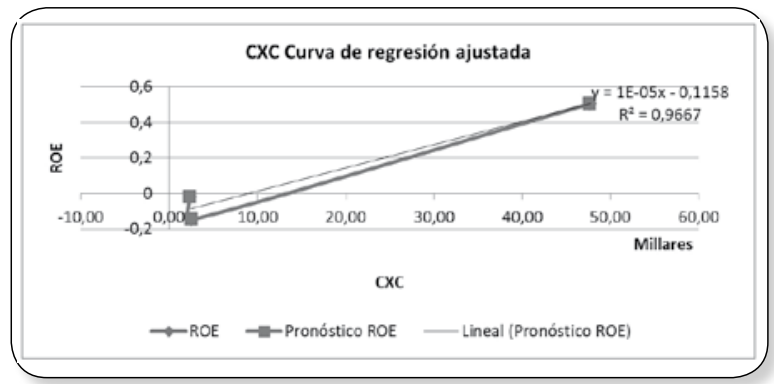

Figura 5. Curva de regresión ajustada KTNO y ROE

Se obtuvo un coeficiente de correlación positivo y el coeficiente de determinación mostró que las CxC explicaron en 96,67\% las variaciones del ROE.

La figura seis muestra la relación entre los Inventarios y ROE

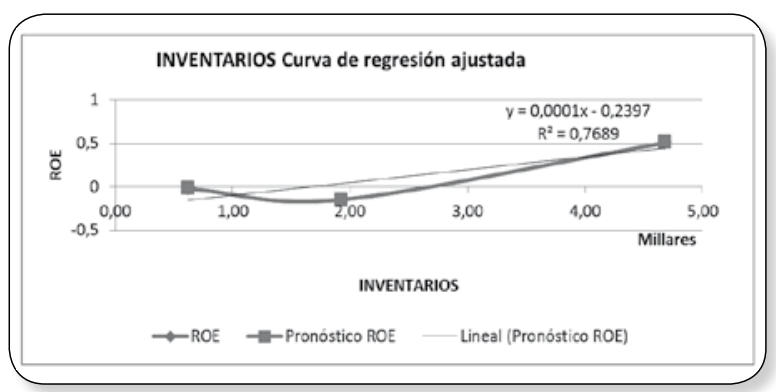

Figura 6. Curva de regresión ajustada KTNO y ROE

Se determinó que los inventarios influyeron en $76,89 \%$ en las variaciones del ROE. En cuanto a las Cuentas por Pagar (CxP) el resultado se muestra en la figura siete

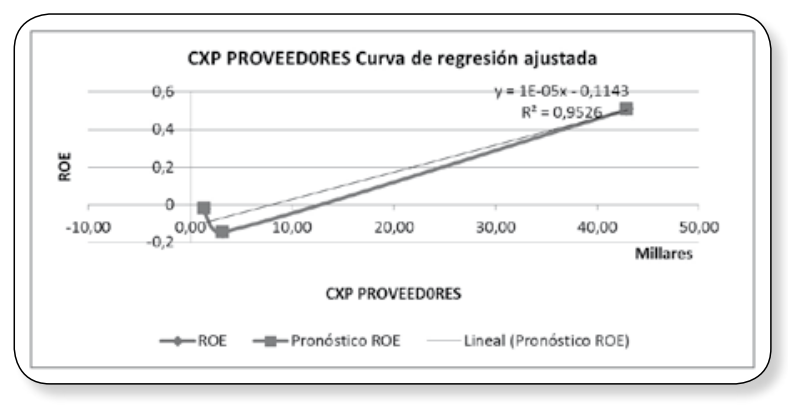

Figura 7. Curva de regresión ajustada $\mathrm{CxP}$ y $\mathrm{ROE}$

Se encontró que las CxP explican en 95,26\% las variaciones del ROE. 
A continuación en la figura 8 se presenta la relación entre la liquidez y $\mathrm{ROE}$

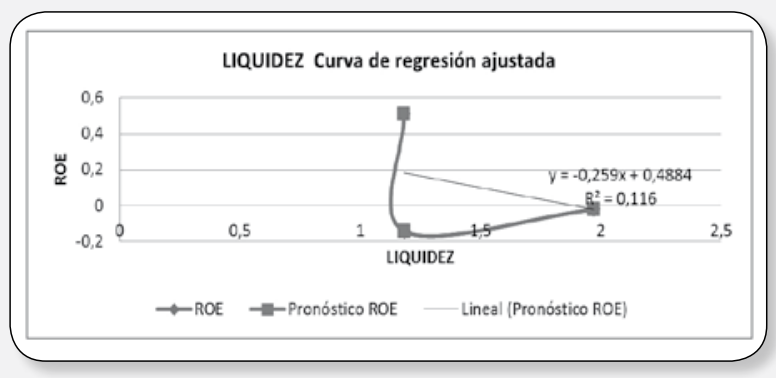

Figura 8. Curva de regresión ajustada Liquidez y ROE

Se determinó que la liquidez explica en un $11,6 \%$ las variaciones del ROE.

También se revisaron otros trabajos sobre la administración del capital de trabajo para conocer los resultados obtenidos. Al respecto se cita a Morales (2011)."El capital de trabajo de las empresas de la industria de la transformación de la Bolsa Mexicana de Valores ante la crisis 2008-2010" Concluyó que las empresas del sector industrial de transformación mantuvieron una política de capital de trabajo ante la crisis económicafinanciera considerada en términos de la teoría como política promedio, caracterizada por niveles en los renglones de activos circulantes en cantidades muy similares a los existentes antes de la crisis de 2008, de tal manera que ha sido un sector que ha logrado sobrevivir aún durante la crisis económica-financiera.

Igualmente se encontró Arcos y otros (2006). En el trabajo se estimó la eficiencia empresarial de un conjunto de sociedades pertenecientes al sector no financiero de Colombia para el periodo de 2001 al 2004. Los resultados son consecuentes con estudios similares realizados en el exterior. De igual forma, los resultados obtenidos permiten concluir que el ciclo del negocio es fundamental en la gestión financiera de las empresas y debe optimizarse en la medida de lo posible. El efecto del apalancamiento operativo y los gastos financieros, como importante determinante en la rentabilidad de las empresas, no parece tener el efecto esperado en la rentabilidad empresarial
Sánchez y Rangel (2010), concluyen que las estrategias financieras de capital de trabajo utilizadas por las empresas del sector farmacéutico, en el municipio de Riohacha, de acuerdo con los resultados arrojados por su investigación son bastante acertadas en la medida en que le han permitido a estas empresas lograr un lugar competitivo en el mercado y por ende desarrollarse. Los resultados demostraron que el $61 \%$ de las empresas del sector aplican estrategias financieras de activos y pasivos circulantes, esto significa que las empresas que utilizan estrategias financieras para el manejo de su capital de trabajo evidencian un buen manejo de sus recursos.

\section{CONCLUSIONES.}

Los resultados obtenidos en el análisis de la administración del capital de trabajo en el sector textil de Cúcuta en el periodo de estudio se alinearon con los postulados teóricos, en este caso con las posiciones tomadas de Gitman, Albornoz y García. Se cumplió que: alta liquidez menor rentabilidad, bajo ciclo de efectivo mayor rentabilidad, baja relación activo corriente/activo total, mayor rentabilidad, alta relación pasivo corriente/pasivo total, mayor rentabilidad, si PKT tiende a cero hace mas eficiente la administración del KTNO y por ende apunta a mayor rentabilidad.

En el análisis del capital de trabajo en el sector textil de Cúcuta en el periodo de estudio, se encontró que el KTNO presento una caída en el año 2009 respecto al año 2008 cuando se calculó en 9,45millones de pesos y alcanzó en el año 2011 la cifra de 1,69 millones de pesos. Aquí se debe hacer resaltar que esto estuvo acompañado de una drástica caída en las ventas lo que afectó los ingresos operacionales. No obstante el año 2011 presentó un incremento respecto al año 2009 pero ante la disminución de los ingresos puede interpretarse como ineficiencia en la gestión. Se verificó que las $\mathrm{CxC}$ tienen un peso aproximado del $45 \%$ en el KTNO y las $\mathrm{CxP}$ un peso similar, correspondiendo a los inventarios el $10 \%$ restante. Esto hizo suponer
Enero - Junio 2014

ISSN 0122-820X

PP: 86-98 
No. 1

Enero - Junio 2014 ISSN 0122-820X

PP: 86-98 que se colocó la mercancía de inventario pero no se efectuaron mas compras o cambiaron las condiciones de financiamiento de los proveedores. La PKT desmejora en cada año de estudio, puede argumentarse que la desmejora de este indicador refleja ineficiencia en la gestión.

Al identificar el comportamiento de la liquidez en el sector textil de Cúcuta en el periodo de estudio se puede decir que los índices de liquidez y autonomía mostraron para el sector textil confecciones en el periodo de estudio valores teóricamente aceptables en el año 2011 se observó que los indicadores venían mejoraron desde el año 2008 y 2009 años en que estuvieron por debajo del índice referencial. En lo que respecta al FCL este presentó un comportamiento variable en el periodo de estudio, en el año 2011 el FCL fue negativo lo que pudo comprometer la gestión financiera del sector.

Cuando se determinó la rentabilidad del sector textil en el periodo de estudio se pudo observar que los componentes de la rentabilidad desmejoran a medida que transcurre el periodo. La rotación disminuye al igual que el margen que se ve afectado por las perdidas en las utilidades antes de intereses e impuestos (BAII), esto llevó a que la rentabilidad del activo o rentabilidad económica fuese negativa en los años 2009 y 2011. En cuanto a la rentabilidad financiera también desmejoró. En conjunto al determinar la Rentabilidad del Patrimonio (ROE), se determinó que pasó del $50,71 \%$ en el año 2008 a una rentabilidad de $-2,07 \%$ en el año 2011, lo que se convierte en una debilidad que debe enfrentar en el sector.

96 Por su parte la rentabilidad del activo neto no benefician al sector ya que es negativa, esto se debe igualmente a la caída de las ventas del sector. Se determinó que durante los años 2008 y 2009 el MEBITDA fue positivo pero en el año 2011 se tornó negativo, es decir demandó caja por cada unidad vendida, llevando esto a consumir recursos del sector.

Al relacionar el capital de trabajo y la rentabilidad en el sector textil de Cúcuta en el periodo de estudio se verificó que el coeficiente de correlación lineal de Pearson se encontró que el KTNO y el ROE presentó un coeficiente de correlación que se puede considerar muy alto positivo al igual que con el RAN. Se determinó que las variaciones del KTNO explican en un $89,4 \%$ las variaciones de la RAN y en un $98,1 \%$ las variaciones del ROE, se estableció la importancia de la adecuada gestión del KTNO como factor de criticidad en la gestión operativa del sector textil confecciones.

$\mathrm{Al}$ relacionar el capital de trabajo, la liquidez y la rentabilidad en el sector textil de Cúcuta periodo 2008-2011 se determinó que la liquidez y el KTNO mostraron un coeficiente de correlación lineal de Pearson negativo de -0,4678 el cual se puede considerar de valor medio, esto significa que las variables se mueven en sentido contrario con una variación conjunta moderada, mientras que el coeficiente de determinación R2 indicó que las variaciones de la liquidez explicarían en un 21,89\% las variaciones del KTNO.

Al analizar la influencia de cada uno de los componentes del KTNO en el ROE se determinó que las CxC explicarían el 96,67\% de las variaciones del ROE mientras que los inventarios lo harían en un 76,89\% y las CxP explicarían en el 95,26\%, se concluye que debe tener especial cuidado el manejo de estos indicadores en la gestión del KTNO. Al analizar la influencia de la liquidez en las variaciones del $\mathrm{ROE}$ se determinó que alcanzaría el 11,6\%, siendo este factor el de menor influencia de los analizados.

Con base en los resultados del estudio se demuestra, que el comportamiento de los componentes del KTNO influye en el comportamiento de la liquidez y de la rentabilidad de una manera asincrónica tal como lo expresaron Cabrera y Amero.

Se corrobora lo planteado por los autores consultados en cuanto a la importancia de la administración del capital de trabajo como factor crítico de éxito en la gestión financiera ya que los resultados obtenidos mostraron alta relación entre las variables e indicadores financieros analizados. 
Al contrastar con otras investigaciones se encontró que los hallazgos de la presente investigación se corresponden con los resultados encontrados en cuanto al cumplimiento de los postulados teóricos de la administración del capital de trabajo.

\section{BIBLIOGRAFÍA}

Agenda Interna para la Productividad y la Competitividad. Documento regional, Norte de Santander. Departamento Nacional de Planeación. Bogotá, junio 2007. Consultado en Mayo 2013 disponible en https://www.mincomercio.gov.co/ptp/descargar.php?id=40564

Albornoz, César. (2006). "Capital de trabajo un tema importante para considerar en las empresas". Publicaciones Universo Económico, UE № 78- Febrero 2006. Buenos Aires. Disponible en http://www.consejo.org.ar/publicaciones/ue/ue78/trabajo.htm consultado en Marzo 2014.

Amaya, I (2009). "Sector textil amenaza cerrar exportaciones a Venezuela" disponible en http://www.vanguardia.com/historico/30982sector-textil-amenaza-cerrar-exportacionesa-venezuela\#sthash.SKIOSGLH.dpuf consultado en Julio 2013.

Arcos y Benavides. (2006). "Efecto del Ciclo de Efectivo Sobre la Rentabilidad de las Firmas Colombianas". Borradores de Economía y Finanzas. No. 9, Diciembre 2006. Gestión editorial Departamento de Economía - Universidad ICESI Cali. Colombia

Ávila Baray, H. L. Introducción a la metodología de la investigación. Disponible en http://www.eumed.net/libros/2006c/203/1u. htm. Consulta: Mayo de 2013

-Balestrini, M. (1998 y 2002). Como se elabora el proyecto de investigación. (2da. Ed.). Bl Consultores asociados. Caracas, Venezuela.

Bernal, Cesar A. (2000). "Metodología de la investigación para. Administración y Economía". Pearson Educación de Colombia. Santafé de Bogotá. Colombia.
Cabrera, R y Amero, A. (2006)."Optimización del Capital de Trabajo más que una razón financiera, un enfoque integral de gestionar la liquidez de la empresa. http:// www.kpmg.com/MX/es/IssuesAndInsights/ ArticlesPublications/Documents/D

E2008/05capitaltrabajo.pdf consultado en Julio 2013

Empresa e Industria Comisión Europea. Industria textil y de la confección. Disponible en http://ec.europa.eu/enterprise/sectors/ textiles/index es.htm Consultado en Diciembre de 2012

Faxas del Toro y Atucha Fuentes: El análisis financiero del capital de trabajo en la empresa, en Observatorio de la Economía Latinoamericana, № 152, 2011. Texto completo en http://www.eumed.net/cursecon/ ecolat/cu/2011/

Gitman, Lawrence. (2003). Principios de Administración Financiera., 10 $\mathrm{a}^{\mathrm{a}}$ Edición. Pearson Educación. México. 676 p

Hernández, Fernández \& Baptista, (2003). Metodología de la Investigación. México: McGraw - Hill.

Disponible en http://www.tec-igital.itcr.ac.cr/ servicios/ojs/index.php/ tec_empresarial/ article/ view/610 consultado en Julio 2013.

Informe Sector Textil y Confecciones Colombiano Análisis Sectorial. (2010). MAPFRE.Crediseguro SA. Medellín.

"La pymes y los empresarios" disponible en (http://asopymescolombia.org/blog/la-pymesy-los-empresarios/es.) consultado en Mayo 2013

Méndez C. (1998). Metodología, guía para elaborar diseños de investigación en ciencias económicas, contables y administrativas ( $2^{\underline{a}}$ ed.). Santa Fe Bogotá: McGraw-Hill.

Morales J., Castro A., Díaz C, López K. (2011). El capital de trabajo de las empresas de la industria de la transformación de la Bolsa Mexicana de Valores ante la crisis 2008-
Enero - Junio 2014

ISSN 0122-820X

PP: $86-98$ 
No. 1

Enero - Junio 2014 ISSN 0122-820X

PP: $86-98$

Moyer, McGuigan, Kretlow, (2005), Administración financiera contemporánea, México, Thomson.

Restrepo, Aníbal y Vanegas, (2010, mayoagosto). Competitividad y comercio exterior de las Pymes del sector textil-confecciones del Valle de Aburrá: incidencia del tipo de cambio. Revista Virtual Universidad Católica del Norte, (30). Recuperado el 12 de Julio de 2013, de: http://revistavirtual.ucn.edu.co/ index.php/RevistaUCN/article/view/63

Rizo L, Solís P: "La teoría del capital de trabajo y sus técnicas" en Contribuciones a la Economía, junio 2010, consultado en http:// www.eumed.net/ce/2010a/ recuperado en Julio 2013

Sánchez, Deusenith; Rangel, Olivia. (2010). "Estrategias financieras de capital de trabajo en empresas farmacéuticas". Artículo de investigación en Libre Empresa Vol. 7 No. 1, Enero - Junio de 2010 pp. 54-67

Valerio D; Segura J; Gutiérrez N. (2011) Análisis de Creación de Valor Económico para Empresas del Sector Textil (Value added analysis for the textile sector businesses). Vol. 5, Núm. 1, 2011. Publicado en TEC empresarial. Escuela de Administración de Empresas del Tecnológico de Costa Rica Disponible en: http://www.tec-digital.itcr. ac.cr/servicios/ojs/index.php/tec empresarial/ article/view/610 consultado en Julio 2013.

Van Horne, James C. y Wachowicz, Jr., John M. J. (1994). Fundamentos de Administración Financiera. Octava Edición. México: Prentice-Hall Hispanoamericana SA. 